\title{
A new system for detection of thermoluminescence and delayed luminescence from photosynthetic apparatus with precise temperature control
}

\author{
Takumi Noguchi ${ }^{\mathrm{a}, \mathrm{b}, *}$, Masaya Katoh ${ }^{\mathrm{a}}$ and Yorinao Inoue ${ }^{\mathrm{a}}$ \\ ${ }^{a}$ RIKEN (The Institute of Physical and Chemical Research), Wako, Saitama 351-0198, Japan \\ ${ }^{\mathrm{b}}$ Institute of Materials Science, University of Tsukuba, Tsukuba, Ibaraki 305-8573, Japan
}

\begin{abstract}
A new system for thermoluminescence (TL) and delayed luminescence (DL) measurements with precise temperature control was developed. A freezing/heating stage for microscope was modified to afford a fixed sample temperature and a constant heating rate. A valve equipped with a low-flow bypass for cold $\mathrm{N}_{2}$ gas through liquid $\mathrm{N}_{2}$ was used for both fine control of the sample temperature and for rapid cooling of the sample after flash illumination. High performance of the system was demonstrated by measurements of the TL glow curves with various heating rates and the DL decays at different temperatures arising from recombination of charged pairs in photosystem II membranes.
\end{abstract}

\section{Introduction}

Thermoluminescence (TL) is emission of light that is observed when irradiated materials are heated. The emission arises from thermally activated recombination of trapped charges at luminescence centers. The temperature at which TL emission occurs reflects the energetic depth of the traps. TL from plant materials was first reported by Arnold and Sherwood [1]. Since then, TL has been extensively used to investigate the properties of charged pairs stabilized in photosynthetic apparatus. In particular, measurement of TL glow curves, the intensity profiles of emission against temperature, in photosystem II (PSII) is a useful probe for monitoring the intermediates (S-states) of the oxygen-evolving Mn-cluster and the redox states of the primary and secondary quinone electron acceptors $\left(\mathrm{Q}_{\mathrm{A}}\right.$ and $\mathrm{Q}_{\mathrm{B}}$, respectively) $[2,3]$. Delayed luminescence (DL), which is observed at a fixed temperature, occurs by basically the same process of charge recombination as TL, and thus measurement of DL decay curves at different temperatures gives similar information as that of TL glow curves. Various analyses of TL glow curves have been proposed to estimate the thermodynamic parameters (e.g., $\Delta G, \Delta H, \Delta S$ ) of the charged pairs [4-10] based on Randall-Wilkins theory [11] or its developed treatments.

The key points of the system for TL measurements of photosynthetic material are (i) fixing the sample temperature before illumination, (ii) freezing the sample quickly after illumination, (iii) heating the sample at a constant heating rate, and (iv) effective collection of emission onto a detector. Since the peak temperature and the shape of a TL glow curve are significantly dependent on the heating rate $[6,12]$,

\footnotetext{
*Corresponding author: Takumi Noguchi, Institute of Materials Science, University of Tsukuba, Tsukuba, Ibaraki 305-8573, Japan. Tel.: +81 29853 5126; Fax: +81 29855 7440; E-mail: tnoguchi@ ims.tsukuba.ac.jp.
} 
keeping it constant during the measurement is particularly important for obtaining data suitable for accurate theoretical analysis. Also, fixing the sample temperature precisely is crucial for DL measurements. In this study, we have developed a new system for TL and DL measurements capable of accurate temperature control. We have applied this system to measure TL glow curves of PSII membranes with various heating rates and also DL decays at various temperatures.

\section{Materials and methods}

The oxygen-evolving PSII membranes [13] were prepared from spinach following the method by Ono and Inoue [14]. The membranes were suspended in a buffer including $40 \mathrm{mM}$ Mes, $400 \mathrm{mM}$ sucrose, $20 \mathrm{mM} \mathrm{CaCl}_{2}, 20 \mathrm{mM} \mathrm{NaCl}_{2}$, and $30 \%(\mathrm{v} / \mathrm{v})$ glycerol $(\mathrm{pH} 6.5)$ at a concentration of $0.3 \mathrm{mg}$ chlorophyll/ml. The membrane suspension also included $1 \mu \mathrm{M}$ 3-(3,4-dichlorophenyl)-1,1-dimethylurea (DCMU) to block the electron transfer from $\mathrm{Q}_{\mathrm{A}}$ to $\mathrm{Q}_{\mathrm{B}}$.

\section{Instrumentation}

Figure 1 shows the block diagram of the system for TL and DL measurements (A) and the expanded view of the sample unit (B). The sample unit is formed by modifying the freezing/heating stage for microscope (LINKAM, TH-600RH). In this freezing/heating stage, the heat block is heated by a sheath heater and is cooled by cold $\mathrm{N}_{2}$ gas that is passed through liquid $\mathrm{N}_{2}$ (Fig. 1B). The temperature of the stage is detected by a platinum resistance thermometer buried in the heat block and monitored by a temperature controller, which in turn determines the voltage to the heater to control the temperature and the heating rate. An aluminum sample holder $(20 \mathrm{~mm} \varnothing ; 1 \mathrm{~mm}$ in thickness) with a hollow $(15 \mathrm{~mm} \varnothing$; $0.5 \mathrm{~mm}$ in depth) was placed on the stage. A sample solution $(85 \mu \mathrm{l})$ is placed in the hollow and covered by an acrylic plate. When the sample is a leaf or a piece of filter paper that absorbs sample solution, it is directly placed on the stage and covered by an acryl plate. The sample temperature is monitored with a digital multi-thermometer (Advantest, TR-2114H) using a sheath thermocouple (type T; $0.5 \mathrm{~mm} \varnothing$ ), which is inserted into the sample solution or pressed against the leaf or filter paper. The flow rate of cold $\mathrm{N}_{2}$ gas is controlled by a valve with a low-flow bypass (CKD, AMD31-15-12-3). When the sample temperature is fixed or raised at a certain heating rate, the valve is closed and the low-flow bypass is used. Rapid cooling of the sample after illumination is done by opening the valve using an electric switch to obtain a high flow rate. The sample unit is purged by $\mathrm{N}_{2}$ gas to avoid frosting.

Light illumination on the sample is performed by flash light with duration of $\sim 4 \mu$ s from a Xe-flash source (Sugawara, SL-230S) controlled by a stroboscope (Sugawara, MS-230). The flash light was led to the sample using a ring-type light guide (HOYA-SCHOTT, FGR6F1000D18R). Emission from the sample is collected by a light guide (16 mm $\varnothing$ ) (HOYA-SCHOTT, LGS16F1000D18-72E), which is fitted into the inner hole of the ring-type light guide and placed above the sample at a close distance of $\sim 7 \mathrm{~mm}$ (Fig. 1B). At the other end of the light guide, the emitted light is focused onto a cooled photomultiplier (Hamamatsu, R943-02). Electromagnetic shutters (Copal, EC-601) are placed in front of the Xe lamp and the photomultiplier, and the former shutter is opened whereas the latter is closed during flash illumination by a trigger from a homemade shutter driver. The signal from the photomultiplier is processed by a photon counting unit (Hamamatsu, C3866) and is transmitted to a personal computer through a photon counting board (Hamamatsu, M3949). This emission data together with the temperature 
data from the digital thermometer are used to produce TL glow curves or DL decays in the computer using a homemade program.
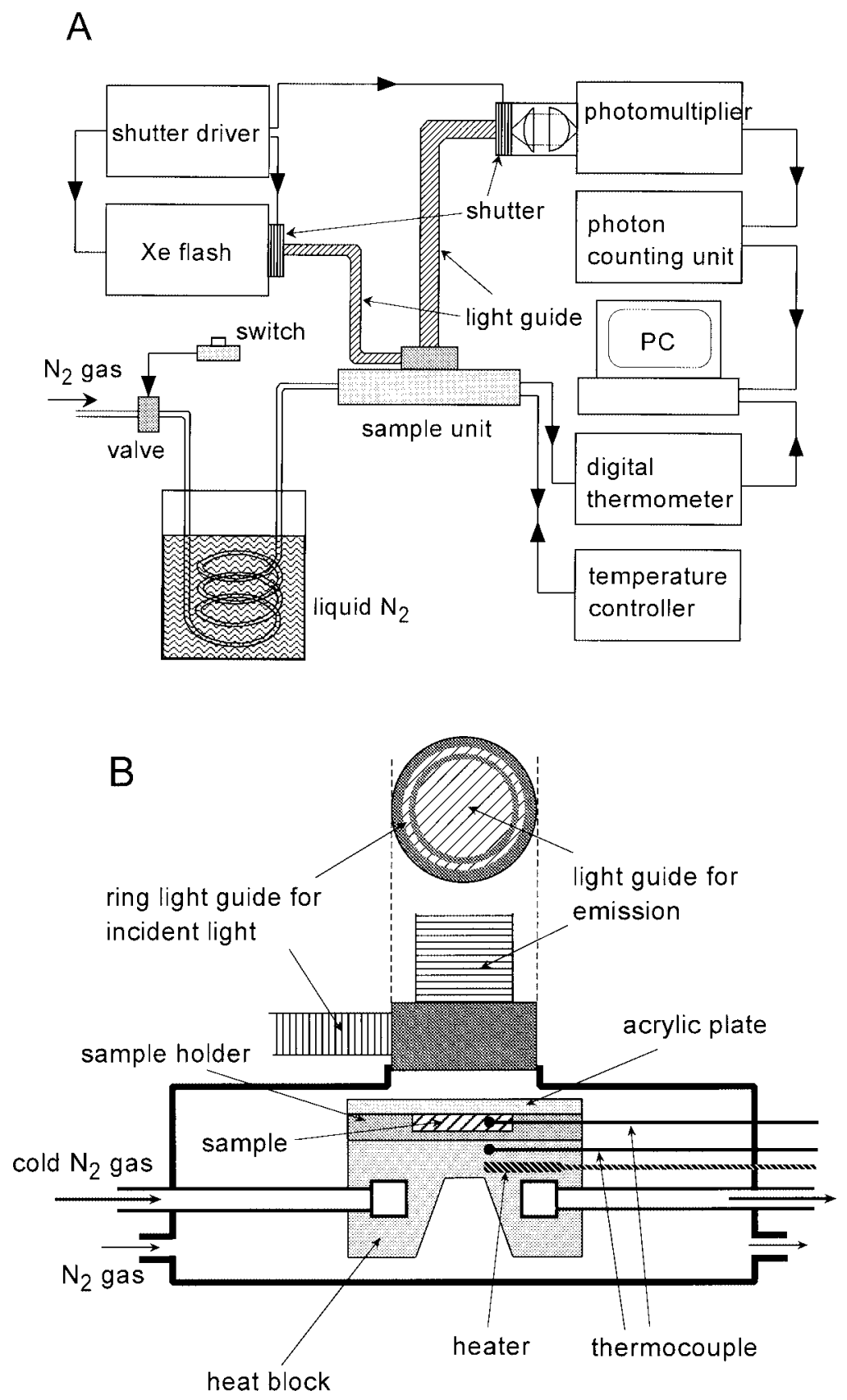

Fig. 1. (A) Block diagram of the system for TL and DL measurements. (B) Expanded view of the sample unit. 


\section{Results and discussion}

Using the above system, TL glow curves of PSII membranes from spinach were measured at various heating rates. The PSII sample suspended in a buffer was excited with a single flash at $5^{\circ} \mathrm{C}$ and immediately frozen by opening the valve for the cold $\mathrm{N}_{2}$-gas line. The sample temperature decreased by about $10^{\circ} \mathrm{C}$ in $3 \mathrm{~s}$ after opening the valve and reached $-180^{\circ} \mathrm{C}$ in $30 \mathrm{~s}$. Upon single-flash illumination of PSII, charge separation takes place at the primary electron donor chlorophyll, $\mathrm{P}_{680}$, and an electron is transferred to $\mathrm{Q}_{\mathrm{A}}$ to yield a $\mathrm{Q}_{\mathrm{A}}^{-}$radical. Further electron transfer to $\mathrm{Q}_{\mathrm{B}}$ is blocked by the inhibitor, DCMU. On the other hand, a hole is transferred to the Mn-cluster and the dark-stable $S_{1}$ state is converted to the $S_{2}$ state. Thus, the charged pair of $\mathrm{S}_{2} \mathrm{Q}_{\mathrm{A}}^{-}$is produced in the PSII sample. This charged pair is stabilized by quick freezing of the sample. The frozen sample was then heated up at a constant heating rate. Figure 2 shows the time courses of the sample temperature at different heating rates. It is seen that the temperature increases linearly at every heating rate.

Figure 3 shows TL glow curves for the $\mathrm{S}_{2} \mathrm{Q}_{\mathrm{A}}^{-}$recombination measured using different heating rates. Note that TL intensities were corrected so that the total emission is proportional to the area of the TL band. The TL peak temperature decreased from 18 to $-4^{\circ} \mathrm{C}$ as the heating rate decreased from 90 to $10^{\circ} \mathrm{C} / \mathrm{min}$. In addition, the TL intensity dramatically decreased with lowering the heating rate. This drastic change in the TL intensity indicates that there is a pathway of non-radiative recombination in PSII and this non-radiative pathway makes an appreciable contribution to the recombination process of $\mathrm{S}_{2} \mathrm{Q}_{\mathrm{A}}^{-}$at least under usual conditions of TL measurements. Thus, it may be necessary to take the nonradiative pathway into account to estimate accurate thermodynamic parameters in theoretical analysis of TL glow curves.

Figure 4 shows the DL decay curves of the PSII membranes upon single-flash illumination measured at -10 (solid line), 0 (dotted line), 10 (dashed line) and $20^{\circ} \mathrm{C}$ (dash-dotted line). Each curve could be fitted with a linear combination of three exponential decays. The slowest decay with a largest contribution (60$70 \%$ ) was assigned to the $\mathrm{S}_{2} \mathrm{Q}_{\mathrm{A}}^{-}$recombination. The estimated time constant of the $\mathrm{S}_{2} \mathrm{Q}_{\mathrm{A}}^{-}$recombination was $66,34,11$ and $6 \mathrm{~s}$ at $-10,0,10$, and $20^{\circ} \mathrm{C}$, respectively. The total emission by this recombination

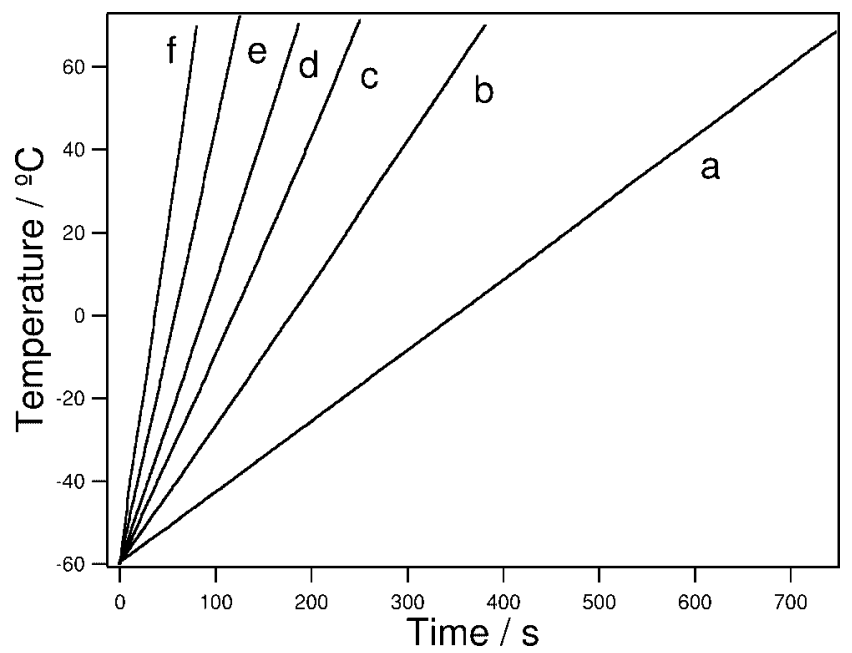

Fig. 2. Time course of the sample temperature during TL measurements with heating rates of $10^{\circ} \mathrm{C} / \mathrm{min}$ (a), $20^{\circ} \mathrm{C} / \mathrm{min}(\mathrm{b}$ ), $30^{\circ} \mathrm{C} / \min (\mathrm{c}), 40^{\circ} \mathrm{C} / \min (\mathrm{d}), 60^{\circ} \mathrm{C} / \mathrm{min}(\mathrm{e}), 90^{\circ} \mathrm{C} / \mathrm{min}$ (f). The sample was PSII membranes suspended in a buffer including $30 \%$ glycerol. 


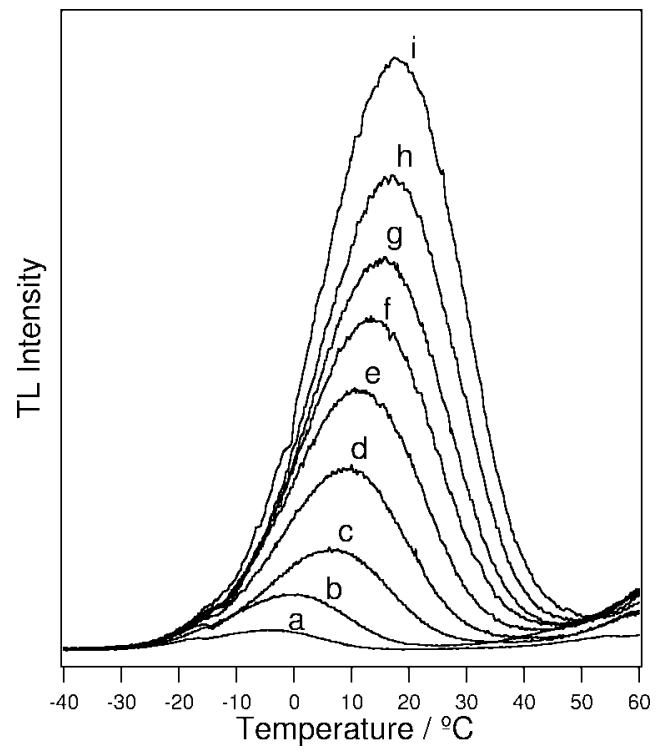

Fig. 3. TL glow curves of PSII membranes in the presence of DCMU after single-flash illumination. The TL signal arises from recombination between $\mathrm{Q}_{\mathrm{A}}^{-}$and the $\mathrm{S}_{2}$ state of the oxygen-evolving Mn-cluster. Illumination on the sample was performed at $5^{\circ} \mathrm{C}$. The heating rates for TL measurements were $10^{\circ} \mathrm{C} / \mathrm{min}$ (a), $20^{\circ} \mathrm{C} / \mathrm{min}$ (b), $30^{\circ} \mathrm{C} / \mathrm{min}$ (c), $40^{\circ} \mathrm{C} / \mathrm{min}$ (d), $50^{\circ} \mathrm{C} / \mathrm{min}$ (e), $60^{\circ} \mathrm{C} / \min (\mathrm{f}), 70^{\circ} \mathrm{C} / \mathrm{min}(\mathrm{g}), 80^{\circ} \mathrm{C} / \mathrm{min}(\mathrm{h})$, and $90^{\circ} \mathrm{C} / \mathrm{min}(\mathrm{i})$.

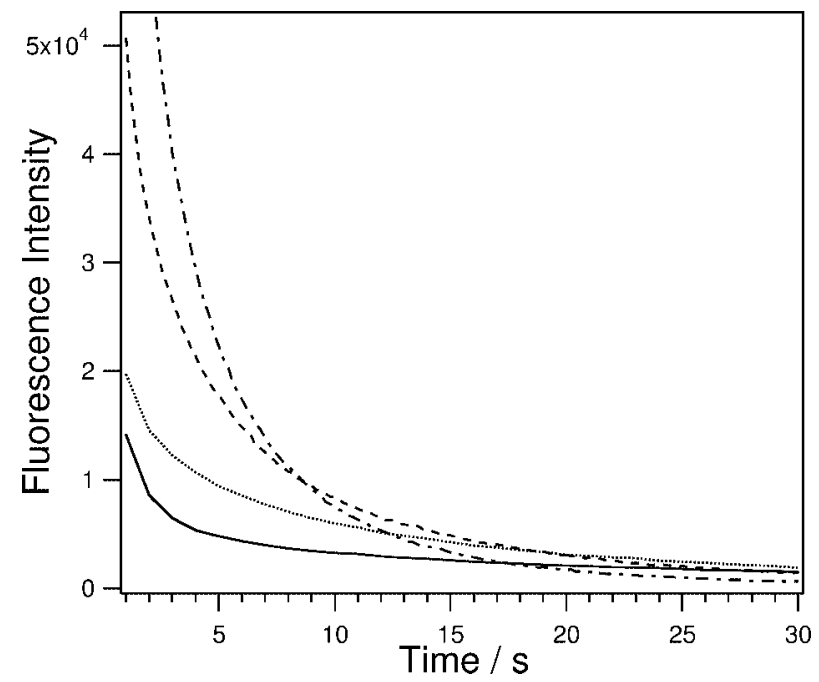

Fig. 4. DL decays of PSII membranes in the presence of DCMU upon single-flash illumination measured at $-10^{\circ} \mathrm{C}$ (solid line), $0^{\circ} \mathrm{C}$ (dotted line), $10^{\circ} \mathrm{C}$ (dashed line) and $20^{\circ} \mathrm{C}$ (dash-dotted line).

decreased by a factor of two at $-10^{\circ} \mathrm{C}$ compared to that at $20^{\circ} \mathrm{C}$, being consistent with the TL result that an appreciable amount of the charged pair decays through a non-radiative pathway. The above time constants confirm that the initial freezing rate of this system $\left(\sim-10^{\circ} \mathrm{C}\right.$ per $\left.3 \mathrm{~s}\right)$ is fast enough to stabilize most of the flash-induced $\mathrm{S}_{2} \mathrm{Q}_{\mathrm{A}}^{-}$pair with minimized loss.

In conclusion, we have developed a new system for TL and DL measurements capable of precise temperature control. High performance of this system was demonstrated by measuring the TL glow 
curves and DL decays of the $\mathrm{S}_{2} \mathrm{Q}_{\mathrm{A}}^{-}$charged pair in PSII membranes from spinach. The TL and DL data obtained by this system will be suitable for theoretical calculations to estimate accurate thermodynamic parameters.

\section{References}

[1] W.A. Arnold and H.K. Sherwood, Are chloroplasts semiconductors?, Proc. Natl. Acad. Sci. USA 43 (1957), $105-114$.

[2] I. Vass and Govindjee, Thermoluminescence from the photosynthetic apparatus, Photosynth. Res. 48 (1996), 117-126.

[3] Y. Inoue, Photosynthetic thermoluminescence as a simple probe of photosystem II electron transport, in: Biophysical Techniques in Photosynthesis, J. Ametz and A.J. Hoff, eds, Kluwer Academic Publishers, Dordrecht, The Netherlands, 1996, pp. 93-107.

[4] I. Vass, G. Horváth, T. Herczeg and S. Demeter, Photosynthetic energy conservation investigated by thermoluminescence. Activation energies and half-lives of thermoluminescence bands of chloroplasts determined by mathematical resolution of glow curves, Biochim. Biophys. Acta 634 (1981), 140-152.

[5] V.G. Tatake, T.S. Desai, Govindjee and P.V. Sane, Energy storage states of photosynthetic membranes: Activation energies and lifetimes of electrons in the trap states by thermoluminescence method, Photochem. Photobiol. 33 (1981), 243-250.

[6] D. DeVault, Govindjee and W. Arnold, Energetics of photosynthetic glow peaks, Proc. Natl. Acad. Sci. USA 80 (1983), 983-987.

[7] D. DeVault and Govindjee, Photosynthetic glow peaks and their relationship with the free energy changes, Photosynth. Res. 24 (1990), 175-181.

[8] P.S. Mazumdar, S.J. Singh and R.K. Gartia, The determination of activation energy from the shape of a thermoluminescence peak, J. Phys. D: Appl. Phys. 21 (1988), 815-819.

[9] J.-M. Ducruet and T. Miranda, Graphical and numerical analysis of thermoluminescence and fluorescence $\mathrm{F}_{0}$ emission in photosynthetic material, Photosynth. Res. 33 (1992), 15-27.

[10] A.V. Klevanik, Thermoluminescence during photosynthesis. II. Applicability of Randall-Wilkins theory, Mol. Biol. 29 (1995), 378-381.

[11] J.T. Randall and M.H.F. Wilkins, Phosphorescence and electron traps. I. The study of trap distributions, Proc. R. Soc. London, Ser. A 184 (1945), 366-389.

[12] G. Kitis, M. Spiropulu, J. Paradopoulos and S. Charalambous, Heating rate effects on the TL glow-peaks of three thermoluminescence phosphors, Nucl. Instr. Meth. B 73 (1993), 367-372.

[13] D.A. Berthold, G.T. Babcock and C.F. Yocum, A highly resolved, oxygen-evolving photosystem II preparation from spinach thylakoid membranes, FEBS Lett. 134 (1981), 231-234.

[14] T. Ono and Y. Inoue, Effects of removal and reconstitution of the extrinsic 33, 24 and $16 \mathrm{kDa}$ proteins on flash oxygen yield in photosystem II particles, Biochim. Biophys. Acta 850 (1986), 380-389. 


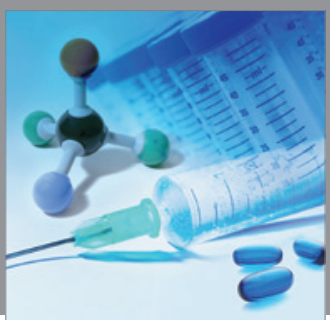

International Journal of

Medicinal Chemistry

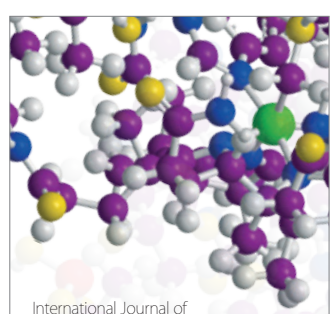

Carbohydrate Chemistry

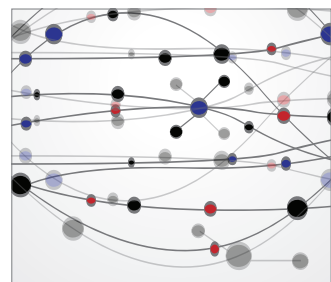

The Scientific World Journal
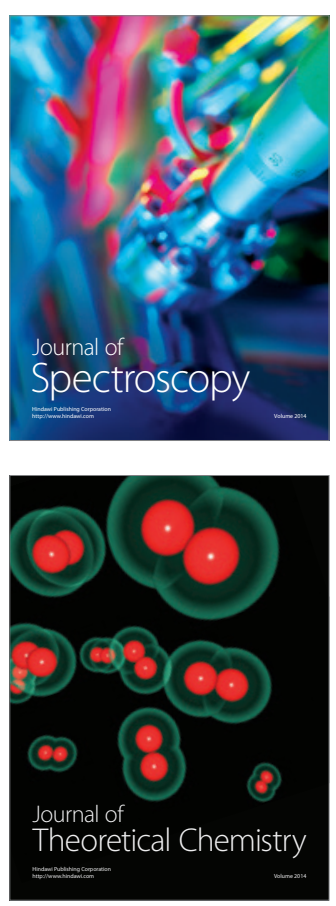
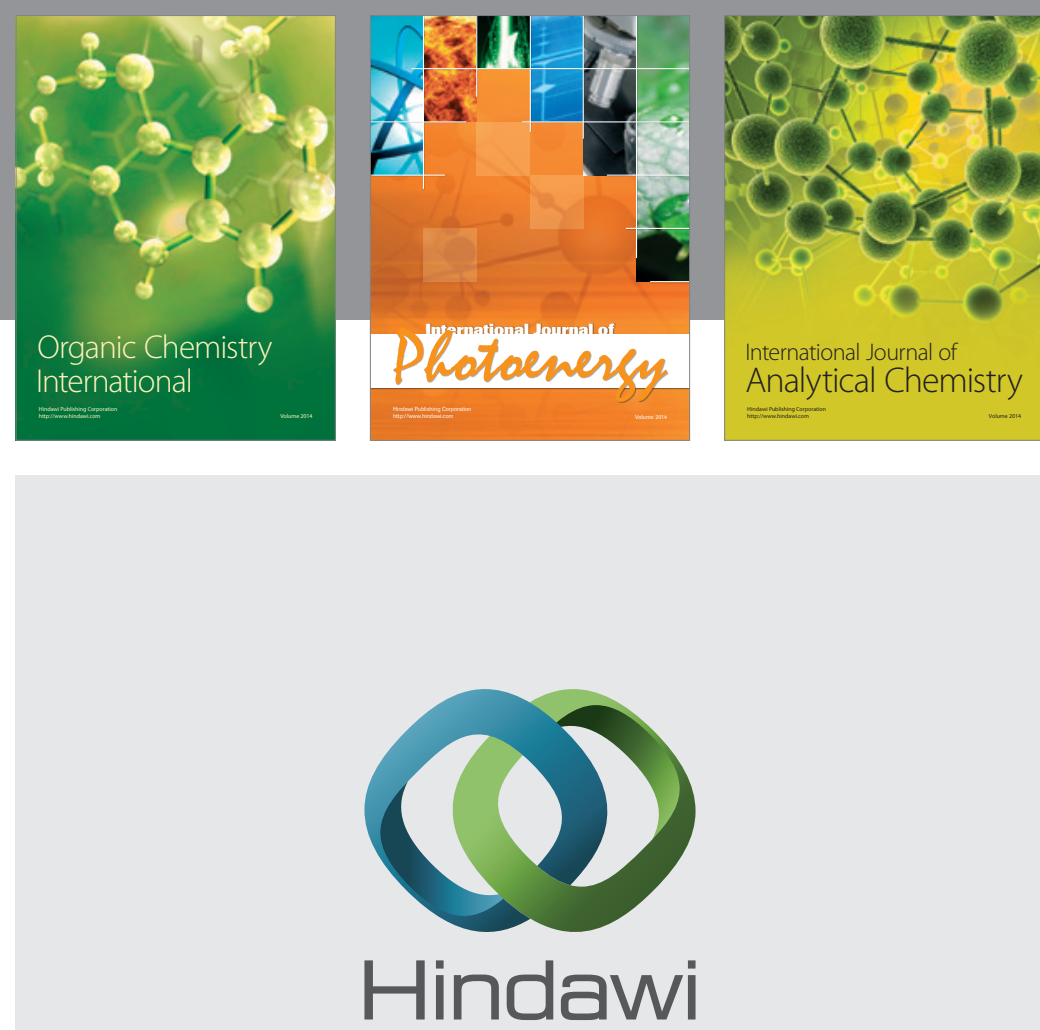

Submit your manuscripts at

http://www.hindawi.com
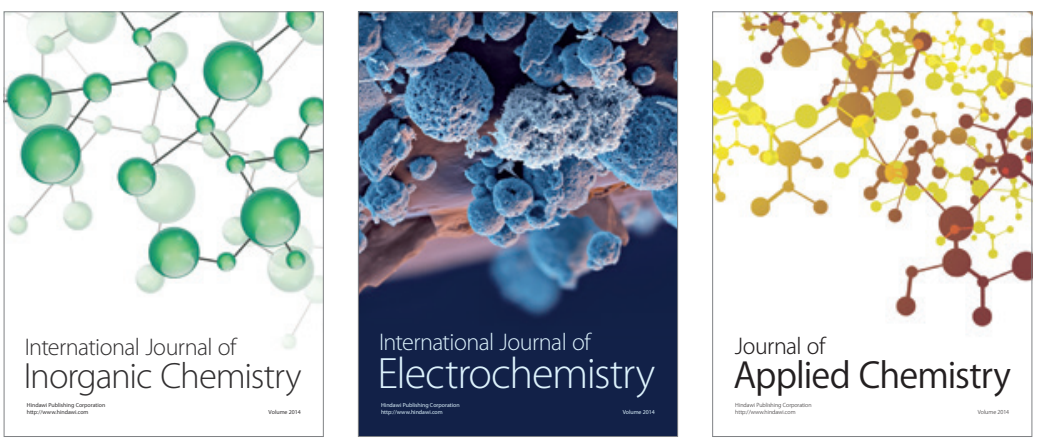

Journal of

Applied Chemistry
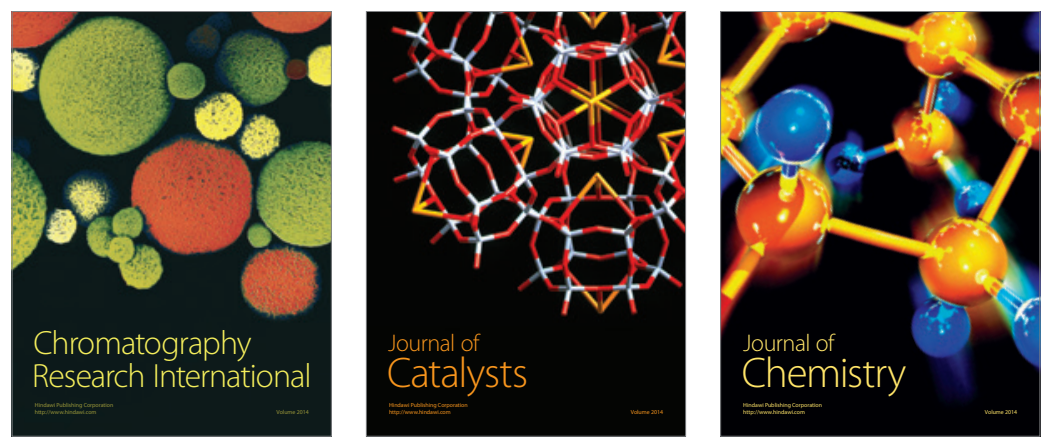
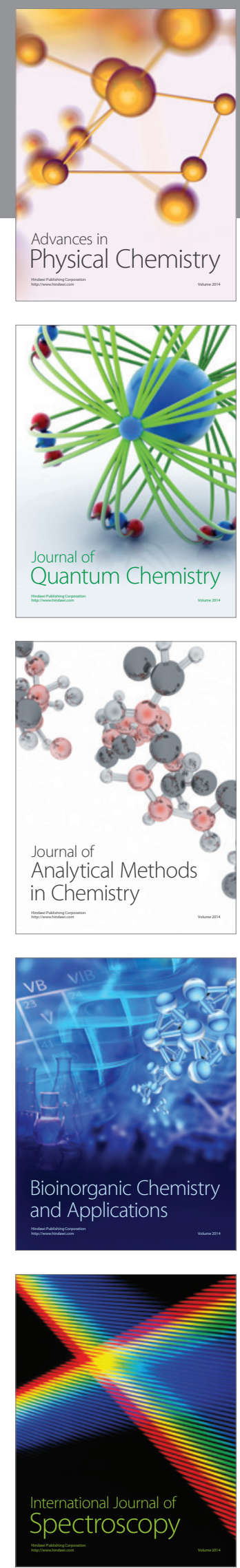